\title{
HEAVY TAILS OF DISCOUNTED AGGREGATE CLAIMS IN THE CONTINUOUS-TIME RENEWAL MODEL
}

\author{
QIHE TANG, ${ }^{*}$ The University of Iowa
}

\begin{abstract}
We study the tail behavior of discounted aggregate claims in a continuous-time renewal model. For the case of Pareto-type claims, we establish a tail asymptotic formula, which holds uniformly in time.
\end{abstract}

Keywords: Asymptotics; extended regular variation; renewal process; uniformity

2000 Mathematics Subject Classification: Primary 91B30

Secondary 60G55; 60G70

\section{Introduction and the main result}

Consider a continuous-time renewal model, in which claim sizes $X_{k}, k=1,2, \ldots$, constitute a sequence of independent, identically distributed (i.i.d.), and nonnegative random variables with common distribution $F$, while their arrival times $\tau_{k}, k=1,2, \ldots$, constitute a renewal counting process,

$$
N_{t}=\#\left\{k=1,2, \ldots: \tau_{k} \leq t\right\}, \quad t \geq 0 .
$$

We assume that $\left\{X_{k}, k=1,2, \ldots\right\}$ and $\left\{N_{t}, t \geq 0\right\}$ are mutually independent. To avoid triviality, we mention that $X_{1}$ and $\tau_{1}$ are not degenerate at zero. We allow $\tau_{1}$ to potentially have a positive probability at zero, not for practical usefulness but for theoretical completeness. Suppose that there is a constant interest force $\delta>0$. That is to say, after time $t$ one dollar becomes $\mathrm{e}^{\delta t}$ dollars. Then, the aggregate claims form into a stochastic process of the form

$$
A_{\delta}(t)=\sum_{k=1}^{\infty} X_{k} \mathrm{e}^{\delta\left(t-\tau_{k}\right)} 1_{\left(\tau_{k} \leq t\right)}, \quad t \geq 0,
$$

where for an event $E$ the symbol $1_{E}$ denotes its indicator function. Since $A_{\delta}(t) \rightarrow \infty$ almost surely as $t \rightarrow \infty$, we instead study the tail behavior of the discounted process,

$$
D_{\delta}(t)=\sum_{k=1}^{\infty} X_{k} \mathrm{e}^{-\delta \tau_{k}} 1_{\left(\tau_{k} \leq t\right)}, \quad t \geq 0 .
$$

We shall derive for the tail probability of $D_{\delta}(t), t \geq 0$, an asymptotic formula, which holds uniformly for all $t$ for which the renewal function,

$$
\lambda_{t}=\mathrm{E} N_{t}=\sum_{k=1}^{\infty} \operatorname{Pr}\left(\tau_{k} \leq t\right)
$$

\footnotetext{
Received 16 October 2006; revision received 8 February 2007.

* Postal address: Department of Statistics and Actuarial Science, The University of Iowa, 241 Schaeffer Hall, Iowa City, IA 52242, USA. Email address: qtang@stat.uiowa.edu
} 
is positive. For this purpose, we define $\Lambda=\left\{t: \lambda_{t}>0\right\}$. With $\underline{t}=\inf \left\{t: \lambda_{t}>0\right\}=$ $\inf \left\{t: \operatorname{Pr}\left(\tau_{1} \leq t\right)>0\right\}$, it is clear that

$$
\Lambda= \begin{cases}{[\underline{t}, \infty]} & \text { if } \operatorname{Pr}\left(\tau_{1}=\underline{t}\right)>0 \\ (\underline{t}, \infty] & \text { if } \operatorname{Pr}\left(\tau_{1}=\underline{t}\right)=0\end{cases}
$$

We shall assume that the distribution $F$ on $[0, \infty)$ is extended-regularly-varying tailed and hence, heavy tailed. That is to say, $\bar{F}(x)=1-F(x)>0$ holds for all $x \geq 0$ and there are some constants $\alpha$ and $\beta, 0<\alpha \leq \beta<\infty$, such that

$$
v^{-\beta} \leq \liminf _{x \rightarrow \infty} \frac{\bar{F}(v x)}{\bar{F}(x)} \leq \limsup _{x \rightarrow \infty} \frac{\bar{F}(v x)}{\bar{F}(x)} \leq v^{-\alpha} \quad \text { for all } v \geq 1 .
$$

We use $F \in \operatorname{ERV}(-\alpha,-\beta)$ to signify the regularity property in (1.4). The class ERV is the union of all classes $\operatorname{ERV}(-\alpha,-\beta)$ over the range $0<\alpha \leq \beta<\infty$. This class has been used in the study of precise large deviations by many people since the work of Klüppelberg and Mikosch (1997). It is well known that ERV is a subclass of the class $\&$ of subexponential distributions; see Theorem 1 of Goldie (1978). The subexponentiality of a distribution $F$ is characterized by the relations $\bar{F}(x)>0$ for all $x \geq 0$ and

$$
\lim _{x \rightarrow \infty} \frac{\overline{F * F}(x)}{\bar{F}(x)}=2 .
$$

Clearly, the class ERV covers the famous class $\mathcal{R}$ of distributions with regularly-varying tails characterized by the relations $\bar{F}(x)>0$ for all $x \geq 0$ and

$$
\lim _{x \rightarrow \infty} \frac{\bar{F}(v x)}{\bar{F}(x)}=v^{-\alpha} \quad \text { for some } \alpha>0 \text { and all } v \geq 1 .
$$

It is usually easier to handle distributions from the class $\mathcal{R}$ because of the well-developed Karamata theory. Although the class ERV is marginally larger than the class $\mathcal{R}$, we expect that asymptotic results for the ERV case provide greater insight into the study of the subexponential case. For more details of heavy-tailed distributions, the reader is referred to Bingham et al. (1987) and Embrechts et al. (1997).

Hereafter, all limit relationships hold for $x$ tending to $\infty$ unless stated otherwise. For two positive functions $a(\cdot)$ and $b(\cdot)$, we write $a(x) \sim b(x)$ if $\lim a(x) / b(x)=1$. Furthermore, for two positive bivariate functions $a(\cdot ; \cdot)$ and $b(\cdot ; \cdot)$, we say that the asymptotic relation $a(x ; t) \sim$ $b(x ; t)$ holds uniformly over all $t$ in a nonempty set $\Delta$ if

$$
\lim _{x \rightarrow \infty} \sup _{t \in \Delta}\left|\frac{a(x ; t)}{b(x ; t)}-1\right|=0 .
$$

Asymptotic formulae that hold with such a uniformity feature are usually of higher theoretical and practical interests.

Recall (1.2) and (1.3). Our main result is given below.

Theorem 1.1. Consider the renewal model introduced above. If $F \in \mathrm{ERV}$, then the relation

$$
\operatorname{Pr}\left(D_{\delta}(t)>x\right) \sim \int_{0-}^{t} \bar{F}\left(x \mathrm{e}^{\delta s}\right) \mathrm{d} \lambda_{s}
$$

holds uniformly for all $t \in \Lambda$. 


\section{Some remarks}

Remark 2.1. When $t=\infty$, the sum $D_{\delta}(t)$ reduces to

$$
D_{\delta}(\infty)=\sum_{k=1}^{\infty} X_{k} \mathrm{e}^{-\delta \tau_{k}}
$$

For $F \in \operatorname{ERV}(-\alpha,-\beta)$, from (3.1) below with $x$ fixed, we see that $\bar{F}(y)=O\left(y^{-\alpha^{\prime}}\right)$ for all $\alpha^{\prime}$, $0<\alpha^{\prime}<\alpha$. Hence, E $X_{1}^{\alpha^{*}}<\infty$ for all $\alpha^{*}, 0<\alpha^{*}<\alpha$. Using this fact we may further verify that $\mathrm{E}\left(D_{\delta}(\infty)\right)^{\alpha^{*} \wedge 1}<\infty$. This means that $D_{\delta}(t)$ converges almost surely as $t$ tends to $\infty$. Likewise, using the fact that $\mathrm{E} X_{1}^{\alpha^{*}}<\infty$ and the elementary renewal theorem, it is easy to verify that $\int_{0-}^{\infty} \bar{F}\left(\mathrm{e}^{\delta s}\right) \mathrm{d} \lambda_{s}<\infty$, irrespective of whether or not $\tau_{1}$ has a finite mean. Therefore, both sides of (1.6) are well defined.

Remark 2.2. Suppose that premiums are collected continuously at a constant rate $c>0$. Then, the surplus process is

$$
S_{\delta}(t)=x \mathrm{e}^{\delta t}+c \int_{0}^{t} \mathrm{e}^{\delta(t-s)} \mathrm{d} s-A_{\delta}(t), \quad t \geq 0,
$$

where $x \geq 0$ denotes the initial surplus. Define the probability of ruin by time $t$ as the probability that the surplus process ever becomes negative by time $t$. Denote this probability by $\psi_{\delta}(x, t)$. The limit $\psi_{\delta}(x, \infty)=\lim _{t \rightarrow \infty} \psi_{\delta}(x, t)$ represents the probability of ultimate ruin. Although the practical relevance of ruin probabilities is questionable, they do provide a good risk measure for insurance business. Clearly, for all $t \in \Lambda$,

$$
\psi_{\delta}(x, t)=\operatorname{Pr}\left(\inf _{0<v \leq t} S_{\delta}(v)<0\right)=\operatorname{Pr}\left(\sup _{0<v \leq t}\left(D_{\delta}(v)-c \int_{0}^{v} \mathrm{e}^{-\delta s} \mathrm{~d} s\right)>x\right) .
$$

Hence,

$$
\psi_{\delta}(x, t) \leq \operatorname{Pr}\left(D_{\delta}(t)>x\right) \quad \text { and } \quad \psi_{\delta}(x, t) \geq \operatorname{Pr}\left(D_{\delta}(t)>x+\frac{c}{\delta}\right) .
$$

Note that, by (1.4), $\bar{F}\left((x+c / \delta) \mathrm{e}^{\delta s}\right) \sim \bar{F}\left(x \mathrm{e}^{\delta s}\right)$ holds uniformly for all $s \in[0, \infty)$. Applying Theorem 1.1, we immediately obtain that the relation

$$
\psi_{\delta}(x, t) \sim \int_{0-}^{t} \bar{F}\left(x \mathrm{e}^{\delta s}\right) \mathrm{d} \lambda_{s}
$$

holds uniformly for all $t \in \Lambda$. Klüppelberg and Stadtmüller (1998) first obtained a result similar to (2.2) with $t=\infty$ for the special case when $\left\{N_{t}, t \geq 0\right\}$ is a homogeneous Poisson process and $F$ belongs to the class $\mathcal{R}$. For this special case, Tang (2005) obtained the uniformity of (2.2). Recently, Chen and $\mathrm{Ng}$ (2007) extended the asymptotic relation (2.2) with $t=\infty$ to the case of negatively dependent claims.

Remark 2.3. We stated Theorem 1.1 in terms of the renewal model, where the innovations $X_{k}, k=1,2, \ldots$, denote claim sizes and hence, are nonnegative. However, in most situations considered in practice, these innovations appearing in (1.2) could be real valued. For this more general case, we may instead study the tail behavior of the running maximum process,

$$
\widetilde{D_{\delta}}(t):=\sup _{0 \leq s \leq t} D_{\delta}(s), \quad t \geq 0 .
$$


We show that the asymptotic formula

$$
\operatorname{Pr}\left(\widetilde{D_{\delta}}(t)>x\right) \sim \int_{0-}^{t} \bar{F}\left(x \mathrm{e}^{\delta s}\right) \mathrm{d} \lambda_{s}
$$

holds uniformly for all $t \in \Lambda$ as long as the right tail of $F$ is still extended regularly varying as described in (1.4). In fact, for this case, the part until (4.3) of the proof given in Subsection 4.1 is valid for both $D_{\delta}(t)$ and $D_{\delta}^{+}(t):=\sum_{k=1}^{\infty} X_{k}^{+} \mathrm{e}^{-\delta \tau_{k}} 1_{\left(\tau_{k} \leq t\right)}, t \geq 0$, where $X_{k}^{+}=X_{k} 1_{\left(X_{k} \geq 0\right)}$ denotes the positive part of $X_{k}$ for $k=1,2, \ldots$. As for all $t \geq 0$,

$$
D_{\delta}(t) \leq \widetilde{D_{\delta}}(t) \leq D_{\delta}^{+}(t)
$$

we see that (2.3) holds uniformly for all $t \in \Lambda \cap[0, T]$ for an arbitrarily fixed number $T \in \Lambda$. The remaining proof of the uniformity on $\Lambda$ of (2.3) can be given by simply copying the part of the proof of Theorem 1.1 given after (4.3) with all the $D_{\delta}$ terms replaced by $\widetilde{D}_{\delta}$ terms.

Remark 2.4. Relation (1.6) unfortunately involves the renewal function $\lambda_{t}, t \geq 0$, as do (2.2) and (2.3). If the i.i.d. interarrival times have a common finite mean $\mathrm{E} \tau_{1}=1 / \lambda$, then $\lambda_{t} \sim \lambda t$ as $t \rightarrow \infty$ by the elementary renewal theorem. This tempts us to consider the replacement of $\lambda_{s}$ in (1.6) by $\lambda s$. However, this is not feasible in general. In fact, under the condition $F \in \mathrm{ERV}$, the differential $\mathrm{d} \lambda_{s}$ in the integral is on an equal footing. Thus, we cannot ignore an integral part in the right neighborhood of zero.

If $\left\{N_{t}, t \geq 0\right\}$ is a homogeneous Poisson process with intensity $\lambda>0$, then $\lambda_{s}=\lambda s$ for all $s>0$. Other cases where the explicit form of the renewal function $\lambda_{s}$ is available can be found in the literature. For example, let $\tau_{1}$ have a phase-type distribution (of which the Erlang distribution is a special case) with density given by

$$
g(s)=\alpha \mathrm{e}^{T s} \boldsymbol{t}, \quad s \geq 0,
$$

where $\boldsymbol{\alpha}$ is a row vector, $\boldsymbol{T}$ is a matrix, and $\boldsymbol{t}=-\boldsymbol{T} \mathbf{1}$ with $\mathbf{1}=(1, \ldots, 1)^{\top}$. The vector $\boldsymbol{\alpha}$ and the matrix $\boldsymbol{T}$ should be chosen such that $(\boldsymbol{\alpha}, 0)$ is the initial distribution and

$$
\left(\begin{array}{ll}
T & t \\
\mathbf{0} & 0
\end{array}\right)
$$

is the intensity matrix of a continuous-time Markov jump process with finite state space in which one state is absorbing and the others are transient. In this case, the derivative of the renewal function $\lambda_{s}$, called the renewal density, is given by

$$
\frac{\mathrm{d} \lambda_{s}}{\mathrm{~d} s}=\boldsymbol{\alpha} \mathrm{e}^{(\boldsymbol{T}+\boldsymbol{t} \boldsymbol{\alpha}) s} \boldsymbol{t} .
$$

As another example, let $\tau_{1}$ have a uniform distribution on $(0, a)$. Then, the renewal density is given by

$$
\frac{\mathrm{d} \lambda_{s}}{\mathrm{~d} s}=\frac{1}{a} \mathrm{e}^{s / a} \sum_{k: 0 \leq k \leq s / a} \mathrm{e}^{-k} \frac{(k-s / a)^{k}}{k !} .
$$

These formulae are copied from Asmussen (2003, p. 88, 148). 
Remark 2.5. We now propose a result for a general situation, in which the renewal function $\lambda_{s}$ can indeed be simplified to $\lambda s$. Consider the discounted process (1.2). For any $x \geq 0$, denote by

$$
T_{x}=\inf \left\{t: D_{\delta}(t)>x\right\}
$$

the first time when $D_{\delta}(t)$ up-crosses the level $x$, where inf $\varnothing=\infty$ by convention. The following is a corollary of Theorem 1.1, giving explicit approximations for the tail probability of $T_{x}$.

Corollary 2.1. In addition to the assumptions of Theorem 1.1, we assume that the i.i.d. interarrival times have a non-lattice distribution and a finite mean $\mathrm{E} \tau_{1}=1 / \lambda$. Then,

$$
\lim _{t \rightarrow \infty} \limsup _{x \rightarrow \infty} \frac{\operatorname{Pr}\left(t<T_{x}<\infty\right)}{\lambda \int_{t}^{\infty} \bar{F}\left(x \mathrm{e}^{\delta s}\right) \mathrm{d} s}=\lim _{t \rightarrow \infty} \liminf _{x \rightarrow \infty} \frac{\operatorname{Pr}\left(t<T_{x}<\infty\right)}{\lambda \int_{t}^{\infty} \bar{F}\left(x \mathrm{e}^{\delta s}\right) \mathrm{d} s}=1 .
$$

If $F \in \mathcal{R}$ as defined in (1.5) with some $\alpha>0$, then (2.4) can be strengthened to

$$
\lim _{t \rightarrow \infty} \lim _{x \rightarrow \infty} \frac{\operatorname{Pr}\left(t<T_{x}<\infty\right)}{(\lambda / \alpha \delta) \mathrm{e}^{-\alpha \delta t} \bar{F}(x)}=1 .
$$

The proof of Corollary 2.1 is left to Section 4 . From the proof one sees that the same result holds for the case discussed in Remark 2.3.

\section{Lemmas}

Lemma 3.1. Let $F \in \operatorname{ERV}(-\alpha,-\beta)$. Then for any $\alpha^{\prime}$ and $\beta^{\prime}, 0<\alpha^{\prime}<\alpha, \beta<\beta^{\prime}<\infty$, there are positive constants $c_{i}$ and $d_{i}, i=1,2$, such that the inequality

$$
\frac{\bar{F}(y)}{\bar{F}(x)} \leq c_{1}\left(\frac{y}{x}\right)^{-\alpha^{\prime}}
$$

holds whenever $y \geq x \geq d_{1}$, and that the inequality

$$
\frac{\bar{F}(y)}{\bar{F}(x)} \geq c_{2}\left(\frac{y}{x}\right)^{-\beta^{\prime}}
$$

holds whenever $y \geq x \geq d_{2}$.

Proof. This lemma is a consequence of Proposition 2.2.1 of Bingham et al. (1987). In fact, with $f=1 / \bar{F}$ we see that (3.1) and (3.2) above are, respectively, (2.2.1') and (2.2.1) of Bingham et al. (1987).

Lemma 3.2. Consider the renewal process $\left\{N_{t}, t \geq 0\right\}$ defined in (1.1). It holds for all $T \in \Lambda$ and all $v>0$ that

$$
\lim _{x \rightarrow \infty} \sup _{t \in \Lambda \cap[0, T]} \frac{1}{\lambda_{t}} \mathrm{E} N_{t}^{v} 1_{\left(N_{t}>x\right)}=0 .
$$

Proof. Follow the proof of Lemma 5.3 of Tang (2004) with slight modifications.

Lemma 3.3. Let $\left\{X_{1}, \ldots, X_{n}\right\}$ be $n$ i.i.d. random variables with common distribution $F \in \&$. Then for arbitrarily fixed numbers $a$ and $b, 0<a \leq b<\infty$, the relation

$$
\operatorname{Pr}\left(\sum_{k=1}^{n} c_{k} X_{k}>x\right) \sim \sum_{k=1}^{n} \bar{F}\left(\frac{x}{c_{k}}\right)
$$

holds uniformly for all $\left(c_{1}, \ldots, c_{n}\right) \in[a, b] \times \cdots \times[a, b]$.

Proof. See Proposition 5.1 of Tang and Tsitsiashvili (2003). 


\section{Proofs}

\subsection{Proof of Theorem 1.1}

To be more precise, we assume $F \in \operatorname{ERV}(-\alpha,-\beta)$. In the first half of this subsection, we prove that (1.6) holds uniformly for all $t \in \Lambda \cap[0, T]$, for an arbitrarily fixed number $T \in \Lambda$. We split the probability $\operatorname{Pr}\left(D_{\delta}(t)>x\right)$ into two parts as

$$
\operatorname{Pr}\left(D_{\delta}(t)>x\right)=\left(\sum_{n=m+1}^{\infty}+\sum_{n=1}^{m}\right) \operatorname{Pr}\left(\sum_{k=1}^{n} X_{k} \mathrm{e}^{-\delta \tau_{k}}>x, N_{t}=n\right):=I_{1}+I_{2},
$$

where $m$ is a temporarily fixed positive integer.

We first deal with $I_{1}$. Recall that $F \in \operatorname{ERV}(-\alpha,-\beta)$. As mentioned in the proof of Lemma 4.5 of Tang (2005), using a result of Nagaev (1979) we may prove that for an arbitrarily fixed number $v>\beta$, there is some $c_{v}>0$ such that for all $n=1,2, \ldots$ and all $x \geq 0$,

$$
\operatorname{Pr}\left(\sum_{k=1}^{n} X_{k}>x\right) \leq c_{v} n^{v} \bar{F}(x)
$$

Therefore,

$$
I_{1} \leq \sum_{n=m+1}^{\infty} \operatorname{Pr}\left(\sum_{k=1}^{n} X_{k}>x\right) \operatorname{Pr}\left(N_{t}=n\right) \leq c_{v} \bar{F}(x) \mathrm{E} N_{t}^{v} 1_{\left(N_{t}>m\right)}
$$

By (3.2), for some $\beta^{\prime}>\beta$ and all $x \geq d_{2}$, we have

$$
\bar{F}(x) \leq \frac{1}{c_{2}} \mathrm{e}^{\delta T \beta^{\prime}} \bar{F}\left(x \mathrm{e}^{\delta T}\right) .
$$

Hence, by Lemma 3.2, for all $x \geq d_{2}$,

$$
\begin{aligned}
& \liminf _{m \rightarrow \infty} \sup _{t \in \Lambda \cap[0, T]} \frac{I_{1}}{\int_{0-}^{t} \bar{F}\left(x \mathrm{e}^{\delta s}\right) \mathrm{d} \lambda_{s}} \\
& \quad \leq \frac{c_{v}}{c_{2}} \mathrm{e}^{\delta T \beta^{\prime}} \liminf _{m \rightarrow \infty} \sup _{t \in \Lambda \cap[0, T]} \frac{\bar{F}\left(x \mathrm{e}^{\delta T}\right) \mathrm{E} N_{t}^{v} 1_{\left(N_{t}>m\right)}}{\bar{F}\left(x \mathrm{e}^{\delta T}\right) \lambda_{t}}=0 .
\end{aligned}
$$

We now turn to $I_{2}$. Under the condition $N_{t}=n$, all $\tau_{k}$ appearing in $I_{2}$ are not larger than $T$. Using Lemma 3.3, it holds uniformly for all $t \in \Lambda \cap[0, T]$ that

$$
\begin{aligned}
I_{2} & =\sum_{n=1}^{m} \operatorname{Pr}\left(\sum_{k=1}^{n} X_{k} \mathrm{e}^{-\delta \tau_{k}}>x \mid N_{t}=n\right) \operatorname{Pr}\left(N_{t}=n\right) \\
& \sim \sum_{n=1}^{m} \sum_{k=1}^{n} \operatorname{Pr}\left(X_{k} \mathrm{e}^{-\delta \tau_{k}}>x \mid N_{t}=n\right) \operatorname{Pr}\left(N_{t}=n\right) \\
& =\left(\sum_{n=1}^{\infty}-\sum_{n=m+1}^{\infty}\right) \sum_{k=1}^{n} \operatorname{Pr}\left(X_{k} \mathrm{e}^{-\delta \tau_{k}}>x, N_{t}=n\right):=I_{21}-I_{22} .
\end{aligned}
$$


Clearly,

$$
\begin{aligned}
I_{21} & =\sum_{k=1}^{\infty} \sum_{n=k}^{\infty} \operatorname{Pr}\left(X_{k} \mathrm{e}^{-\delta \tau_{k}}>x, N_{t}=n\right) \\
& =\sum_{k=1}^{\infty} \operatorname{Pr}\left(X_{k} \mathrm{e}^{-\delta \tau_{k}}>x, \tau_{k} \leq t\right) \\
& =\int_{0-}^{t} \bar{F}\left(x \mathrm{e}^{\delta s}\right) \mathrm{d} \lambda_{s} .
\end{aligned}
$$

Note that

$$
I_{22} \leq \bar{F}(x) \sum_{n=m+1}^{\infty} n \operatorname{Pr}\left(N_{t}=n\right) .
$$

Hence, similar to (4.1), for all $x \geq d_{2}$,

$$
\lim _{m \rightarrow \infty} \sup _{t \in \Lambda \cap[0, T]} \frac{I_{22}}{\int_{0-}^{t} \bar{F}\left(x \mathrm{e}^{\delta s}\right) \mathrm{d} \lambda_{s}}=0 .
$$

We conclude that the asymptotic relation (1.6) holds uniformly for all $t \in \Lambda \cap[0, T]$.

In the second half of this subsection, we extend the uniformity of (1.6) to the whole interval $\Lambda$. For arbitrarily fixed numbers $\alpha^{\prime}$ and $\beta^{\prime}, 0<\alpha^{\prime}<\alpha, \beta<\beta^{\prime}<\infty$, again by (3.1) and (3.2), it holds that, for all $x \geq \max \left\{d_{1}, d_{2}\right\}$ and all $t \in[0, \infty)$,

$$
\frac{\int_{t}^{\infty} \bar{F}\left(x \mathrm{e}^{\delta s}\right) \mathrm{d} \lambda_{s}}{\int_{0-}^{\infty} \bar{F}\left(x \mathrm{e}^{\delta s}\right) \mathrm{d} \lambda_{s}}=\frac{\int_{t}^{\infty}\left(\bar{F}\left(x \mathrm{e}^{\delta s}\right) / \bar{F}(x)\right) \mathrm{d} \lambda_{s}}{\int_{0-}^{\infty}\left(\bar{F}\left(x \mathrm{e}^{\delta s}\right) / \bar{F}(x)\right) \mathrm{d} \lambda_{s}} \leq \frac{c_{1} \int_{t}^{\infty} \mathrm{e}^{-\delta s \alpha^{\prime}} \mathrm{d} \lambda_{s}}{c_{2} \int_{0-}^{\infty} \mathrm{e}^{-\delta s \beta^{\prime}} \mathrm{d} \lambda_{s}} .
$$

The right-hand side of the above tends to zero as $t$ tends to infinity. Therefore, for any $\varepsilon>0$, there exists some $T_{0} \in \Lambda$ such that the inequality

$$
\int_{T_{0}}^{\infty} \bar{F}\left(x \mathrm{e}^{\delta s}\right) \mathrm{d} \lambda_{s} \leq \varepsilon \int_{0}^{T_{0}} \bar{F}\left(x \mathrm{e}^{\delta s}\right) \mathrm{d} \lambda_{s}
$$

holds for all $x \geq \max \left\{d_{1}, d_{2}\right\}$. Recall (2.1). Using Theorem 3.1 of Tang and Tsitsiashvili (2004), we find that

$$
\operatorname{Pr}\left(D_{\delta}(\infty)>x\right) \sim \sum_{k=1}^{\infty} \operatorname{Pr}\left(X_{k} \mathrm{e}^{-\delta \tau_{k}}>x\right)=\int_{0-}^{\infty} \bar{F}\left(x \mathrm{e}^{\delta s}\right) \mathrm{d} \lambda_{s} .
$$

Hence, (1.6) holds for $t=\infty$.

We are ready to extend the uniformity of (1.6) to the whole interval $\Lambda$. On the one hand, it holds uniformly for all $t \in\left(T_{0}, \infty\right]$ that

$$
\begin{aligned}
\operatorname{Pr}\left(D_{\delta}(t)>x\right) & \geq \operatorname{Pr}\left(D_{\delta}\left(T_{0}\right)>x\right) \\
& \sim \int_{0-}^{T_{0}} \bar{F}\left(x \mathrm{e}^{\delta s}\right) \mathrm{d} \lambda_{s} \\
& \geq\left(\int_{0-}^{t}-\int_{T_{0}}^{\infty}\right) \bar{F}\left(x \mathrm{e}^{\delta s}\right) \mathrm{d} \lambda_{s} \\
& \geq(1-\varepsilon) \int_{0-}^{t} \bar{F}\left(x \mathrm{e}^{\delta s}\right) \mathrm{d} \lambda_{s},
\end{aligned}
$$


where in the second step we used (1.6) with $t$ replaced by $T_{0}$, while in the last step we used (4.3). On the other hand, likewise, it holds uniformly for all $t \in\left(T_{0}, \infty\right]$ that

$$
\begin{aligned}
\operatorname{Pr}\left(D_{\delta}(t)>x\right) & \leq \operatorname{Pr}\left(D_{\delta}(\infty)>x\right) \\
& \sim \int_{0-}^{\infty} \bar{F}\left(x \mathrm{e}^{\delta s}\right) \mathrm{d} \lambda_{s} \\
& \leq\left(\int_{0-}^{t}+\int_{T_{0}}^{\infty}\right) \bar{F}\left(x \mathrm{e}^{\delta s}\right) \mathrm{d} \lambda_{s} \\
& \leq(1+\varepsilon) \int_{0-}^{t} \bar{F}\left(x \mathrm{e}^{\delta s}\right) \mathrm{d} \lambda_{s}
\end{aligned}
$$

where in the second step we used (4.4), while in the last step we used (4.3). Hence, it holds for all $t \in\left(T_{0}, \infty\right]$ and all large $x$, say $x>x_{1}>0$, that

$$
(1-2 \varepsilon) \int_{0-}^{t} \bar{F}\left(x \mathrm{e}^{\delta s}\right) \mathrm{d} \lambda_{s} \leq \operatorname{Pr}\left(D_{\delta}(t)>x\right) \leq(1+2 \varepsilon) \int_{0-}^{t} \bar{F}\left(x \mathrm{e}^{\delta s}\right) \mathrm{d} \lambda_{s} .
$$

From the first half of this proof we see that (4.5) still holds for all $t \in \Lambda \cap\left[0, T_{0}\right]$ and all large $x$, say $x>x_{2}>0$. Therefore, (4.5) holds for all $t \in \Lambda$ and all $x>\max \left\{x_{1}, x_{2}\right\}$. As $\varepsilon>0$ is arbitrary, we have obtained the uniformity of (1.6) over all $t \in \Lambda$.

\subsection{Proof of Corollary 2.1}

As every trajectory of $D_{\delta}(t)$ is piecewise constant with only upward jumps, we have $\operatorname{Pr}\left(T_{x} \leq t\right)=\operatorname{Pr}\left(D_{\delta}(t)>x\right)$ for all $t \in \Lambda \cap[0, \infty)$ and $\operatorname{Pr}\left(T_{x}<\infty\right)=\operatorname{Pr}\left(D_{\delta}(\infty)>x\right)$. Hence, by Theorem 1.1, for all $t \in \Lambda \cap[0, \infty)$,

$$
\begin{aligned}
\operatorname{Pr}\left(t<T_{x}<\infty\right) & =\operatorname{Pr}\left(D_{\delta}(\infty)>x\right)-\operatorname{Pr}\left(D_{\delta}(t)>x\right) \\
& \sim \int_{0-}^{\infty} \bar{F}\left(x \mathrm{e}^{\delta s}\right) \mathrm{d} \lambda_{s}-\int_{0-}^{t} \bar{F}\left(x \mathrm{e}^{\delta s}\right) \mathrm{d} \lambda_{s} \\
& =\int_{t}^{\infty} \bar{F}\left(x \mathrm{e}^{\delta s}\right) \mathrm{d} \lambda_{s}
\end{aligned}
$$

where in the second step we used the asymptotic relation (1.6). There is no problem with this step because, with $t$ arbitrarily fixed, similar to (4.2),

$$
\liminf _{x \rightarrow \infty} \frac{\int_{0-}^{\infty} \bar{F}\left(x \mathrm{e}^{\delta s}\right) \mathrm{d} \lambda_{s}}{\int_{0-}^{t} \bar{F}\left(x \mathrm{e}^{\delta s}\right) \mathrm{d} \lambda_{s}} \geq 1+\frac{c_{2} \int_{t}^{\infty} \mathrm{e}^{-\delta s \beta^{\prime}} \mathrm{d} \lambda_{s}}{c_{1} \int_{0-}^{t} \mathrm{e}^{-\delta s \alpha^{\prime}} \mathrm{d} \lambda_{s}}>1
$$

For any $\ell>0$, by the well-known Blackwell renewal theorem,

$$
\lim _{s \rightarrow \infty}\left(\lambda_{s+\ell}-\lambda_{s}\right)=\lambda \ell
$$

It follows that, for any $\varepsilon>0$ and all large $s$, say $s>s_{1}=s_{1}(\varepsilon, \ell)>0$,

$$
(1-\varepsilon) \lambda \ell \leq \lambda_{s+\ell}-\lambda_{s} \leq(1+\varepsilon) \lambda \ell
$$


Therefore, for all $x \in[1, \infty)$ and $t>s_{1}$,

$$
\begin{aligned}
\int_{t}^{\infty} \bar{F}\left(x \mathrm{e}^{\delta s}\right) \mathrm{d} \lambda_{s} & =\sum_{k=1}^{\infty} \int_{t+(k-1) \ell}^{t+k \ell} \bar{F}\left(x \mathrm{e}^{\delta s}\right) \mathrm{d} \lambda_{s} \\
& \leq \sum_{k=1}^{\infty} \bar{F}\left(x \mathrm{e}^{\delta[t+(k-1) \ell]}\right)(1+\varepsilon) \lambda \ell \\
& \leq(1+\varepsilon) \lambda \sum_{k=1}^{\infty} \int_{t+(k-2) \ell}^{t+(k-1) \ell} \bar{F}\left(x \mathrm{e}^{\delta s}\right) \mathrm{d} s \\
& =(1+\varepsilon) \lambda \int_{t}^{\infty} \bar{F}\left(x \mathrm{e}^{\delta(s-\ell)}\right) \mathrm{d} s .
\end{aligned}
$$

Using the definition in (1.4), it holds for all $x \in[1, \infty)$ and all large $s$, say $s>s_{2}>0$, that

$$
\bar{F}\left(x \mathrm{e}^{\delta(s-\ell)}\right) \leq(1+\varepsilon) \mathrm{e}^{\beta \delta \ell} \bar{F}\left(x \mathrm{e}^{\delta s}\right) .
$$

It follows that, for all $x \in[1, \infty)$ and $t>\max \left\{s_{1}, s_{2}\right\}$,

$$
\int_{t}^{\infty} \bar{F}\left(x \mathrm{e}^{\delta s}\right) \mathrm{d} \lambda_{s} \leq(1+\varepsilon)^{2} \mathrm{e}^{\beta \delta \ell} \lambda \int_{t}^{\infty} \bar{F}\left(x \mathrm{e}^{\delta s}\right) \mathrm{d} s .
$$

A similar lower bound for the integral $\int_{t}^{\infty} \bar{F}\left(x \mathrm{e}^{\delta s}\right) \mathrm{d} \lambda_{s}$ can also be established. Hence, by the arbitrariness of the constants $\ell$ and $\varepsilon$, the relation

$$
\int_{t}^{\infty} \bar{F}\left(x \mathrm{e}^{\delta s}\right) \mathrm{d} \lambda_{s} \sim \lambda \int_{t}^{\infty} \bar{F}\left(x \mathrm{e}^{\delta s}\right) \mathrm{d} s, \quad t \rightarrow \infty,
$$

holds uniformly for all $x \in[1, \infty)$. Clearly, the uniformity of (4.7) indicates that

$$
\lim _{t \rightarrow \infty} \limsup _{x \rightarrow \infty} \frac{\int_{t}^{\infty} \bar{F}\left(x \mathrm{e}^{\delta s}\right) \mathrm{d} \lambda_{s}}{\lambda \int_{t}^{\infty} \bar{F}\left(x \mathrm{e}^{\delta s}\right) \mathrm{d} s}=\lim _{t \rightarrow \infty} \liminf _{x \rightarrow \infty} \frac{\int_{t}^{\infty} \bar{F}\left(x \mathrm{e}^{\delta s}\right) \mathrm{d} \lambda_{s}}{\lambda \int_{t}^{\infty} \bar{F}\left(x \mathrm{e}^{\delta s}\right) \mathrm{d} s}=1 .
$$

From (4.6) and (4.8) we have

$$
\begin{aligned}
\lim _{t \rightarrow \infty} \limsup _{x \rightarrow \infty} \frac{\operatorname{Pr}\left(t<T_{x}<\infty\right)}{\lambda \int_{t}^{\infty} \bar{F}\left(x \mathrm{e}^{\delta s}\right) \mathrm{d} s} & =\lim _{t \rightarrow \infty}\left(\lim _{x \rightarrow \infty} \frac{\operatorname{Pr}\left(t<T_{x}<\infty\right)}{\int_{t}^{\infty} \bar{F}\left(x \mathrm{e}^{\delta s}\right) \mathrm{d} \lambda_{s}} \limsup _{x \rightarrow \infty} \frac{\int_{t}^{\infty} \bar{F}\left(x \mathrm{e}^{\delta s}\right) \mathrm{d} \lambda_{s}}{\lambda \int_{t}^{\infty} \bar{F}\left(x \mathrm{e}^{\delta s}\right) \mathrm{d} s}\right) \\
& =1 .
\end{aligned}
$$

The derivation above with lim sup replaced by lim inf is still valid. This proves (2.4).

Likewise, when $F \in \mathcal{R}$ with some $\alpha>0$,

$$
\begin{aligned}
\lim _{t \rightarrow \infty} \lim _{x \rightarrow \infty} \frac{\operatorname{Pr}\left(t<T_{x}<\infty\right)}{(\lambda / \alpha \delta) \mathrm{e}^{-\alpha \delta t} \bar{F}(x)} & =\lim _{t \rightarrow \infty}\left(\lim _{x \rightarrow \infty} \frac{\operatorname{Pr}\left(t<T_{x}<\infty\right)}{\int_{t}^{\infty} \bar{F}\left(x \mathrm{e}^{\delta s}\right) \mathrm{d} \lambda_{s}} \lim _{x \rightarrow \infty} \frac{\int_{t}^{\infty} \bar{F}\left(x \mathrm{e}^{\delta s}\right) \mathrm{d} \lambda_{s}}{(\lambda / \alpha \delta) \mathrm{e}^{-\alpha \delta t} \bar{F}(x)}\right) \\
& =\lim _{t \rightarrow \infty}\left(\frac{1}{(\lambda / \alpha \delta) \mathrm{e}^{-\alpha \delta t}} \lim _{x \rightarrow \infty} \int_{t}^{\infty} \frac{\bar{F}\left(x \mathrm{e}^{\delta s}\right)}{\bar{F}(x)} \mathrm{d} \lambda_{s}\right) \\
& =\lim _{t \rightarrow \infty}\left(\frac{1}{(\lambda / \alpha \delta) \mathrm{e}^{-\alpha \delta t}} \int_{t}^{\infty} \mathrm{e}^{-\alpha \delta s} \mathrm{~d} \lambda_{s}\right) \\
& =\lim _{t \rightarrow \infty}\left(\frac{1}{(\lambda / \alpha \delta) \mathrm{e}^{-\alpha \delta t}} \int_{t}^{\infty} \mathrm{e}^{-\alpha \delta s} \lambda \mathrm{d} s\right) \\
& =1,
\end{aligned}
$$


where in the second step we used (4.6), in the third step we used the dominated convergence theorem justified by (3.1) and (1.5), and in the fourth step we applied the Blackwell renewal theorem as we did in (4.7). This proves (2.5).

\section{Acknowledgements}

The author wishes to thank the referee for his/her very helpful comments. The support of the Old Gold Summer Fellowship from the University of Iowa is acknowledged.

\section{References}

[1] Asmussen, S. (2003). Applied Probability and Queues. 2nd edn. Springer, New York.

[2] Bingham, N. H., Goldie, C. M. And Teugels, J. L. (1987). Regular variation. Cambridge University Press.

[3] Chen, Y. AND NG, K. W. (2007). The ruin probability of the renewal model with constant interest force and negatively dependent heavy-tailed claims. Insurance Math. Econom. 40, 415-423.

[4] Embrechts, P., Klüppelberg, C. And Mikosch, T. (1997). Modelling Extremal Events for Insurance and Finance. Springer, Berlin.

[5] Goldie, C. M. (1978). Subexponential distributions and dominated-variation tails. J. Appl. Prob. 15, 440-442.

[6] Klüppelberg, C. And Mikosch, T. (1997). Large deviations of heavy-tailed random sums with applications in insurance and finance. J. Appl. Prob. 34, 293-308.

[7] KlüPPELberg, C. AND StadTMüller, U. (1998). Ruin probabilities in the presence of heavy-tails and interest rates. Scand. Actuarial J. 1998, 49-58.

[8] Nagaev, S. V. (1979). Large deviations of sums of independent random variables. Ann. Prob. 7, 745-789.

[9] TANG, Q. (2004). Uniform estimates for the tail probability of maxima over finite horizons with subexponential tails. Prob. Eng. Inf. Sci. 18, 71-86.

[10] TANG, Q. (2005). The finite-time ruin probability of the compound Poisson model with constant interest force. J. Appl. Prob. 42, 608-619.

[11] TAng, Q. AND Tsitsiashvili, G. (2003). Randomly weighted sums of subexponential random variables with application to ruin theory. Extremes 6, 171-188.

[12] TANG, Q. And Tsitsiashvili, G. (2004). Finite- and infinite-time ruin probabilities in the presence of stochastic returns on investments. Adv. Appl. Prob. 36, 1278-1299. 